\title{
INNOVACIÓN ABIERTA, CRECIMIENTO Y RENDIMIENTO EN LA PYME DE LA INDUSTRIA AUTOMOTRIZ DE MÉXICO
}

\author{
Gonzalo Maldonado Guzmán* \\ https://orcid.org/0000-0001-8814-6415 \\ Ricardo García Ramírez** \\ https://orcid.org/0000-0002-6498-1157 \\ Silvia Mata Zamores*** \\ https://orcid.org/0000-0002-9917-2691 \\ María Mónica Castillo Esparza**** \\ https://orcid.org/0000-0003-3330-8741
}

RECIBIDO: Septiembre 2020 / ACEPTADO: Noviembre 2020 / PUBLICADO: Enero 2021

Como citar: Maldonado Guzmán, Gonzalo; García Ramírez, Ricardo; Mata Zamores, Silvia; Castillo Esparza, María. (2021). Innovación abierta, crecimiento y rendimiento en la pyme de la industria automotriz de México. Telos: revista de Estudios Interdisciplinarios en Ciencias Sociales, 23 (1), Venezuela. (Pp.85-99).

DOI: www.doi.org/10.36390/telos231.07

\section{RESUMEN}

En la literatura científica del campo de la innovación, la innovación abierta es un tema relativamente reciente y es considerada como una estrategia empresarial esencial para la obtención de mejores resultados de las empresas. Sin embargo, son escasos los estudios publicados en la literatura que se han orientado en el análisis de la innovación abierta en las pequeñas y medianas empresas, aún y cuando en diversos países este tipo de empresas representan la mayoría de las empresas. En este sentido, el objetivo de este estudio es el análisis y discusión de la relación existente entre la innovación abierta, el crecimiento y el rendimiento de las pequeñas y medianas empresas manufactureras de la industria automotriz de México. La metodología corresponde a una investigación cuantitativa con la aplicación de 300 encuestas a una muestra representativa, y la utilización de un modelo de ecuaciones estructurales. La encuesta contiene 14 indicadores desde la perspectiva teórica de Van de Vrande, De Jong, Vanhaverbeke y De Rochemont (2009), Bag (2014), Linder (2006), Carneiro

\footnotetext{
* Licenciado en Economía, Master en Marketing, Doctor en Marketing por la Universidad de Valencia (España). Profesor del Departamento de Mercadotecnia del Centro de Ciencias Económicas y Administrativas de la Universidad Autónoma de Aguascalientes (México), Secretario de Investigación y Posgrados. Correo electrónico: gmaldona@correo.uaa.mx

** Contador Público, Maestría en Finanzas, Doctor en Ciencias Administrativas por la Universidad Autónoma de Aguascalientes (México). Profesor del Departamento de Finanzas del Centro de Ciencias Económicas y Administrativas de la Universidad Autónoma de Aguascalientes (México). Correo electrónico: rgarciar@correo.uaa.mx

*** Licenciada en Administración, Maestría en Administración, Doctora en Administración por la Universidad Autónoma de Querétaro (México). Profesor del Departamento de Administración del Centro de Ciencias Económicas y Administrativas de la Universidad Autónoma de Aguascalientes (México), Secretaria Técnica del Doctorado en Ciencias Administrativas. Correo electrónico: silvia.mata@correo.uaa.mx
}

**** Licenciada en Administración, estudiante de la Maestría en Administración en el Centro de Ciencias Económicas y Administrativas de la Universidad Autónoma de Aguascalientes. Correo electrónico: mony.castillojm@hotmail.com 
(2007) y Kruger y Johnson (2009). Los conceptos de innovación abierta y rendimiento tienen como Principales referentes teóricos a Brunswicker y Chesbrough (2018), Sivam, Dieguez, Pinto y Silva (2019), y de Erol y Klug (2020). Finalmente, se concluye que la innovación abierta tiene efectos positivos significativos, tanto en el rendimiento empresarial como en el nivel de crecimiento.

Palabras clave: Innovación abierta; innovación; crecimiento; rendimiento empresarial; Pyme; industria automotriz.

\title{
Open innovation, growth and performance in mexican SME automotive industry
}

\begin{abstract}
In the scientific literature in the field of innovation, open innovation is a relatively recent topic and is considered an essential business strategy for obtaining better results from companies. However, there are few studies published in the literature that have focused on the analysis of open innovation in small and medium-sized companies, even though in different countries this type of company represents the majority of companies. In this sense, the objective of this study is the analysis and discussion of the relationship between open innovation, growth and performance of small and medium-sized manufacturing companies in the Mexican automotive industry. The methodology corresponds to a quantitative investigation with the application of 300 surveys to a representative sample, and the use of a model of structural equations. The survey contains 14 indicators from the theoretical perspective of Van de Vrande, De Jong, Vanhaverbeke and De Rochemont (2009), Bag (2014), Linder (2006), Carneiro (2007) and Kruger and Johnson (2009). The concepts of open innovation and performance have as main theoretical references Brunswicker and Chesbrough (2018), Sivam, Dieguez, Pinto and Silva (2019), and Erol and Klug (2020). Finally, it is concluded that open innovation has significant positive effects, both on business performance and on the level of growth.
\end{abstract}

Keywords: Open innovation; innovation; growth; business performance; SME; automotive industry.

\section{Introducción}

En la literatura científica de la innovación, el concepto de innovación abierta es considerado por investigadores, académicos y profesionales de la industria como un constructo que está ganando cada vez más adeptos, ya que comúnmente genera una mayor eficiencia y sustentabilidad a las actividades de innovación de las empresas manufactureras (Chesbrough y Brunswicker, 2014). Además, la innovación abierta implica que los procesos de innovación no sean en su totalidad controlados por las empresas, particularmente por las pequeñas y medianas empresas (Pymes), precisamente porque la innovación abierta requiere el compartir información, conocimiento y habilidades con otras empresas del mismo sector y/o organismos nacionales e internacionales (Erol y Klug, 2020).

De igual manera, generalmente la innovación abierta es analizada y discutida en la literatura científica por investigadores, académicos, profesionales de la industria y responsables políticos (Podmetina, Teplov, Albats y Dabrowska, 2016), pero aun existe un número importante 
de empresas manufactureras que están dudando de los resultados de la implementación de la innovación abierta en sus procesos de innovación (Brunswicker y Chesbrough, 2018). Diferentes factores han sido identificados en la literatura que han limitado la adopción de la innovación abierta en las empresas manufactureras, entre los más importantes se encuentran la falta de una apropiada cultura organizacional y un aprendiza práctico de las actividades de la innovación abierta (Wang, Dunn y Coulton, 2015).

Además, la adopción de la innovación abierta requiere de un esfuerzo complejo por parte de las empresas manufactureras, así como del involucramiento de múltiples actores, ya que es considerada como una de las actividades estratégicas que generan un mayor nivel de crecimiento y rendimiento empresarial (Erol y Klug, 2020). Sin embargo, en la literatura científica se han identificado diversas barreras que frenan la adopción e implementación de la innovación abierta en las empresas manufactureras, particularmente en las Pymes (Chesbrough y Brunswicker, 2014), entre las más importante se encuentran la limitación de los recursos, la cultura empresarial y los elevados costos de las actividades de investigación y desarrollo (Avalos y Hernández, 2019).

Asimismo, la innovación abierta puede ayudar a las Pymes manufactureras a eliminar esas barreras y superar sus limitaciones, al proporcionarles un enfoque diferente que les permita abrir y expandir sus distintas actividades de innovación sin incrementar significativamente sus costos (Erol y Klug, 2020). Sin embargo, aun no queda claro cómo la implementación de la innovación abierta afecta de manera negativa el crecimiento y el rendimiento empresarial, por lo cual hace falta más evidencia empírica al respecto (Hu et al., 2015), por lo cual el objetivo de este estudio es analizar la relación existente entre la innovación abierta, el crecimiento y el rendimiento empresarial de las Pymes de la industria automotriz de México.

\section{Bases Teórico-Conceptuales}

El término de innovación es ampliamente aceptado por los profesionales de la academia y la industria, como un elemento sustancial que permite a las empresas de todos los tamaños y sectores mejorar su nivel de crecimiento y rendimiento empresarial (Sivam, Dieguez, Pinto y Silva, 2019). Además, la innovación es vista como una de las estrategias que permite a las empresas, sobre todo a las Pymes, prosperar, crecer e incrementar sus utilidades (Hungund y Kiran, 2015). Desde este punto de vista, es posible establecer que el crecimiento y el rendimiento empresarial de las empresas manufactureras, particularmente de las Pymes que integran la industria automotriz, depende esencialmente de su habilidad para ser más innovadoras (Triguero, Fernández y Sáez, 2018).

Asimismo, en la literatura científica se asume que la innovación en un concepto demasiado complejo que requiere de cambios multifactoriales en las empresas manufactureras (Sivam et al., 2019), ya que comúnmente es aceptado que la innovación es esencial para lograr un mayor nivel de crecimiento, para la creación de nuevas empresas y para abordar los desafíos sociales a nivel de la empresa, regionales o nacionales (European Commission, 2014). Por ello, la predisposición a la innovación por parte de las Pymes manufactureras, facilitará la obtención de ventajas competitivas permanentes, ya que la innovación deberá articularse a las actividades cotidianas que realizan las Pymes manufactureras para lograr mejores resultados (Rivotti, 2015).

Además, en la literatura científica existen diversas definiciones del concepto de innovación, una de las más aceptadas por la comunidad científica es la propuesta por el Manual 
de Oslo (OECD, 2005). Así, de acuerdo al Manual de Oslo, la innovación puede ser considerada como "la implementación de nuevos o mejorados productos (bienes o servicios), procesos (métodos de producción y suministro), métodos de marketing (embalaje, ventas y distribución), o nuevos métodos de gestión (mejores prácticas, mejorar en el lugar de trabajo, relaciones externas, comercialización)" (OECD, 2005. p.46), que es la definición que se utilizará para efectos de este estudio empírico.

En este sentido, la innovación es importante para las empresas manufactureras, entre ellas las que integran la industria automotriz, no sólo a nivel micro como un elemento esencial que les permita mejorar su nivel de crecimiento y rendimiento empresarial (Maier, Keppler y Maier, 2014), sino también a nivel macro a través de la generación de beneficios para la sociedad y como un elemento esencial para el crecimiento económico (Sag, Sezen y Güzel, 2016). Así, la innovación usualmente es considerada en la literatura científica como un proceso acumulativo de diversas actividades que permiten a las empresas manufactureras definir y solucionar los distintos problemas de participación del mercado (Hu et al., 2015).

\section{Actividades de Colaboración}

En la literatura científica, la innovación es considerada como una actividad básica que tiene lugar al interior de las empresas de manera particular (Sag et al., 2016). Sin embargo, hoy en día es prácticamente imposible que una empresa por sí sola pueda generar todos los recursos necesarios para el desarrollo de las actividades de la innovación (Triguero et al., 2018), ya que los productos que demanda el mercado son cada vez más complejos, por lo cual las empresas, particularmente las Pymes, necesitar integrar el conocimiento existente en el exterior a través de actividades de colaboración con otros empresas y organismo públicos y privados, al intercambiar entre ellas los recursos disponibles, habilidades y conocimientos existentes en cada una de ellas (Schiuma y Carlucci, 2018).

Además, la implementación de la innovación abierta en las Pymes manufactureras a través de la creación de actividades de colaboración, está generando distintas oportunidades de aprendizaje no solamente para las empresas, sino también para los académicos y las autoridades gubernamentales (Erol y Klug, 2020). Asimismo, de entre las diversas instituciones y organizaciones que generalmente colaboran con las Pymes manufactureras, las instituciones académicas son los socios más importantes para mejorar las actividades de innovación (Benneworth, Pinheiro y Karlsen, 2017; Schiuma y Carlucci, 2018), ya que las universidades generan una parte importante del conocimiento que necesitan las Pymes para la adopción e implementación de la innovación abierta (Erol y Klug, 2020).

En este sentido, Schiuma y Carlucci (2018) propusieron un modelo integral del establecimiento y desarrollo de una relación estratégica de las Pymes con las universidades en la implementación de actividades de innovación abierta, ya que generalmente las universidades tienen establecidas una variedad de unidades tales como incubadoras de empresas, centros de emprendimiento y unidades de negocios, que permiten el intercambio de conocimiento entre las instituciones y las Pymes manufactureras (Erol y Klug, 2020). Por ello, la realización de actividades entre las Pymes manufactureras, particularmente con aquellas que integran la industria automotriz, puede incrementar la efectividad del crecimiento y el nivel del rendimiento empresarial (Schiuma y Carlucci, 2018). 
Así, las actividades de colaboración son consideradas en la literatura científica de la innovación, como una parte fundamental de las actividades de la innovación abierta (Chesbrough y Brunswicker, 2014). Por lo tanto, las Pymes manufactureras que implementen la innovación abierta a través de las actividades de colaboración tanto con universidades como con sus principales socios comerciales, tendrán mayor oportunidades no solamente de incrementar significativamente su nivel de ventas y rendimiento empresarial, sino también de definir con claridad lo que la organización quiere, necesita y sus expectativas, lo cual les permitirá incrementar el valor de sus actividades de innovación (de Oliveira, Echeveste, Cortimiglia y Goncéales, 2017).

\section{Innovación Abierta}

En la literatura científica de la innovación, diversos investigadores, académicos y profesionales de la industria han demostrado que la innovación abierta genera tres beneficios sustanciales a las empresas manufactureras, particularmente a las Pymes: intercambio de conocimiento, reducción de riesgos y velocidad en el desarrollo de las actividades de innovación (Heidemann y Timenes, 2017). En efecto, la innovación abierta puede implicar varias características tales como el entrenamiento del personal de las empresas involucradas, la reducción de riesgos, el intercambio de conocimiento e información (Kratzer, Maissner y Rould, 2017), la cual comúnmente genera un incremento sustancial tanto en el nivel de crecimiento como en el rendimiento empresarial (Sivam et al., 2019).

Asimismo, para lograr este tipo de resultados es esencial que las empresas manufactureras, tengan un claro conocimiento de la estructura y los procesos que facilitan la implementación de la innovación abierta a nivel organizacional (Bogers, Zobel, Afuah, Almirall, Brunswicker, Dahlander y Wal, 2017), las estrategias de gestión del conocimiento (Cammarano, Caputo, Lamberti y Michelino, 2017), así como su lado humano (Ahn, Minshall y Mortara, 2017). Sin embargo, en la actual literatura de la innovación abierta se establece que en los estudios publicados no se ha analizado ampliamente la teoría de este constructo (Bogers et al., 2018; Lopes y Carvalho, 2018), las investigaciones no han aportado suficiente evidencia empírica (Randhawa, Wilden y Hohberger, 2016) y la mayoría de los estudios han sido descriptivos (Martinez, Soto y Carayannis, 2017).

Además, el análisis y discusión de la implementación de la innovación abierta en las industrias tradicionales y en las Pymes manufactureras es relativamente escasa (Triguero et al., 2018). Asimismo, tradicionalmente la literatura científica de la innovación abierta se ha orientado en las industrias de alta tecnología (Del Río et al., 2016), dejando de lado prácticamente a las Pymes de las industrias de bajo nivel de tecnología, otros estudio más se han enfocado en las industrias tradicionales utilizando muestras demasiado grandes (Pellegrini, Lazzarotti y Manzini, 2014; Kastelli et al., 2016; Seyfettinoglu, 2016), e incluso la evidencia empírica del análisis de la innovación abierta en las Pymes manufactureras es demasiado limitada (Omta, Fortuin y Dijkman, 2014).

Además, diversos investigadores y académicos han considerado que la innovación abierta genera más beneficios a las Pymes que a las grandes empresas (Sag et al., 2016), ya que la innovación abierta incrementa la capacidad de innovación de las Pymes, y tiene un mayor nivel de importancia para las Pymes que para las grandes empresas (Vahter, Love y Roper, 2014). Asimismo, Vahter et al. (2014) encontraron que las Pymes son menor abiertas a la 
innovación que las grandes empresas en los países en vías de desarrollo, pero es exactamente lo contrario en los países desarrollados, lo cual permite establecer que hace falta más evidencia empírica (Sag et al., 2016).

\section{Innovación Abierta, Crecimiento y Rendimiento Empresarial}

En la literatura científica diversos investigadores, académicos y profesionales de la industria, establecen que la innovación abierta es considerado como uno de los tópicos más significativos de la gestión de la innovación en las distintas empresas manufactureras (Sivam et al., 2019), ya que la apertura de los procesos de innovación es una de las premisas esenciales para la implementación de las actividades de la innovación abierta, la cual genera y transforma el conocimiento en nuevos o mejorados productos o servicios, que son ofertados en los mercados en los cuales participan las empresas manufactureras, fomentando con ello una fuerte cultura empresarial (European Commission, 2016).

Adicionalmente, la innovación abierta requiere de la creación de conocimiento interno significativo, basado en el desarrollo de capacidades que permitan a las Pymes manufactureras adquirir el conocimiento y la tecnología externos existentes para generar nuevos o mejorados productos que les permitan obtener un mayor nivel de crecimiento y rendimiento empresarial (Sag et al., 2016). Sin embargo, la falta de personal que posea las habilidades para el desarrollo de conocimiento interno e integración del conocimiento externo existente en el mercado, hace prácticamente imposible que las Pymes manufactureras puedan lograr los niveles de crecimiento y rendimiento empresarial al implementar la innovación abierta, al igual que el obtenido por las grandes empresas (Dodourova y Bevis, 2014).

En este sentido, Spithoven et al. (2013) argumentó que las Pymes manufactureras comparados con las grandes empresas, efectivamente tienen un menor impacto de las actividades de la innovación abierta en el nivel de crecimiento y rendimiento empresarial, precisamente porque no tienen las mismas capacidades para detectar, asimilar e integrar el conocimiento externo existente en el mercado, pero las Pymes manufactureras, particularmente las que integran la industria automotriz, si pueden hacer uso del conocimiento generado en las organizaciones, ya que la calidad del conocimiento que se genera tanto en las universidades como en los centro de investigación es sustancial, y puede generar una incremento sustancial el nivel de crecimiento y rendimiento empresarial (Sag et al., 2016).

Sin embargo, generalmente las Pymes manufactureras tienen serios problemas en la transformación y explotación del conocimiento generado en las universidades y centros de investigación, precisamente porque carecen del personal capacitado, como si lo tienen las grades empresas (Spithoven et al., 2013). Este tipo de acciones limita la capacidad y habilidad de las Pymes manufactureras de acceder a los recursos externos que les permitan mejorar significativamente su nivel de crecimiento y rendimiento empresarial, comparado con otras organizaciones (Dodourova y Bevis, 2014), por lo cual, en primera instancia, las Pymes requieren de mejorar sus capacidades de gestión como una parte integral del sistema de innovación abierta (Brunswicker y Ehrenmann, 2013).

\section{Materiales y Métodos}

Para dar respuesta al objetivo de investigación, se realizó un estudio empírico en las Pymes manufactureras de la industria automotriz de México, utilizando como marco de 
referencia el directorio empresarial de la industria automotriz de México, el cual tenía registradas 909 empresas hasta el 30 de noviembre de 2018. La encuesta para la recolección de la información se aplicó a una muestra de 460 empresas seleccionadas mediante un muestreo aleatorio simple, con un error máximo del $\pm 4 \%$ y un nivel de confiabilidad del $95 \%$, representando dicha muestra el $50.6 \%$ del total de la población, y aplicándose la encuesta durante los meses de enero a marzo de 2019.

Para la medición de la innovación abierta se realizó una adaptación de la escala propuesta por Van de Vrande, De Jong, Vanhaverbeke y De Rochemont (2009), quienes consideraron que ésta se puede ser medida a través de 7 ítems. Para la medición del rendimiento empresarial se realizó una adaptación a la escala propuesta por Bag (2014), quien midió este constructo a través de 6 ítems. Todos los ítems de las escalas de la innovación abierta y el rendimiento empresarial, fueron medidos a través de una escala tipo Likert de cinco puntos, con $1=$ total desacuerdo a $5=$ total acuerdo como límites, ya que este tipo de medición proporciona un excelente balance entre la complejidad de las respuestas de los entrevistados y el análisis de la información (Bryman, 2016; Forza, 2016; Hair, Celsi, Money, Samouel y Page, 2016).

Asimismo, con respecto a la medición del crecimiento este fue medido a través de las ventas realizadas por las Pymes manufactureras de la industria automotriz en el año 2019 (Linder, 2006; Carneiro, 2007; Kruger y Johnson, 2009), ya que para estimar el potencial de crecimiento que puedan lograr las Pymes manufactureras, generalmente se considera una evaluación cualitativa de los gerentes siendo las ventas el principal indicador para su medición (Kruger y Johnson, 2009). Además, para el análisis de los datos se utilizará el software SmartPLS 3.3, ya que el PLS está desarrollándose como una técnica de modelización de ecuaciones estructurales que se emplea en una variedad de disciplinas (do Valle y Assaker, 2015; Richter, Sinkovics, Ringle y Schlägel, 2016; Richter, Cepeda, Roldán y Ringle, 2016).

Tabla 1. Fiabilidad y validez convergente del modelo de innovación abierta

\begin{tabular}{|l|c|c|c|}
\hline \multicolumn{1}{|c|}{ Variable } & Alfa de Cronbach & $\begin{array}{c}\text { Índice de Fiabilidad } \\
\text { Compuesta (IFC) }\end{array}$ & $\begin{array}{c}\text { Índice de la Varianza } \\
\text { Extraída (IVE) }\end{array}$ \\
\hline Crecimiento & 1.000 & 1.000 & 1.000 \\
\hline Innovación Abierta & 0.920 & 0.936 & 0.677 \\
\hline Rendimiento Empresarial & 0.883 & 0.910 & 0.627 \\
\hline
\end{tabular}

Fuente: Elaboración propia

Los resultados de la aplicación del modelo de ecuaciones estructurales a través del partial least squares (PLS-SEM) se presentan en la Tabla 1, e indican la existencia tanto de fiabilidad, medida a través del Alfa de Cronbach y el IFC, cuyos valores son superiores al valor de 0.7 recomendado, como de validez convergente medida a través del IVE cuyos valores son mayores al valor de 0.5 recomendado, cabe aclarar que el crecimiento tiene el valor de 1 porque solamente se midió a través de un solo ítem. Por ello, es posible establecer que los datos derivados de la aplicación de las escalas de innovación abierta, el crecimiento y el rendimiento empresarial son fiables y válidos para la toma de decisiones (Richter et al., 2016). 
Tabla 2. Validez discriminante del modelo de innovación abierta

\begin{tabular}{|l|c|c|c|}
\hline \multicolumn{1}{|c|}{ Variables } & Crecimiento & Innovación Abierta & $\begin{array}{c}\text { Rendimiento } \\
\text { Empresarial }\end{array}$ \\
\hline Innovación Abierta & 0.040 & & \\
\hline Rendimiento Empresarial & 0.116 & 0.490 & \\
\hline
\end{tabular}

Fuente: Elaboración propia

Con respecto a la validez discriminante de las escalas utilizadas, la Tabla 2 indica que en la aplicación del ratio HTMT (Heterotrait-Monotrait), todos los valores del HTMT están claramente por debajo del umbral más conservador de 0.85 . Por ello, de acuerdo con estos resultados es posible establecer la existencia de validez discriminante entre la innovación abierta, el nivel de crecimiento y el rendimiento empresarial, lo cual nos indica que los tres constructos están midiendo variables distintas, pero que están relacionados entre sí (Henseler, Ringle y Sarstedt, 2015).

\section{Resultados}

Para dar respuesta al objetivo planteado en este estudio se aplicó un PLS-SEM utilizando para ello el software SmartPLS, ya que a través del algoritmo del PLS se estiman los coeficientes path y otros parámetros del modelo de tal manera que se maximiza la varianza explicada de los constructos de la innovación abierta, el nivel de crecimiento y rendimiento empresarial (Bentler y Huang, 2014; Dijkstra, 2014; Dijkstra y Henseler, 2015a, b). Además, la validez nomológica del modelo teórico de la innovación abierta se analizó a través del test de la Chi-cuadrada, mediante el cual se compararon los resultados entre los modelos teórico y de medida obteniendo resultados estadísticamente no significativos, lo cual facilita el análisis de los datos (Sánchez, Trinchera y Russolillo, 2015). La Figura 1 que se presenta a continuación muestra con mayor detalle los resultados obtenidos de la aplicación del PLS-SEM.

Figura 1. Resultados del PLS-SEM del modelo de innovación abierta

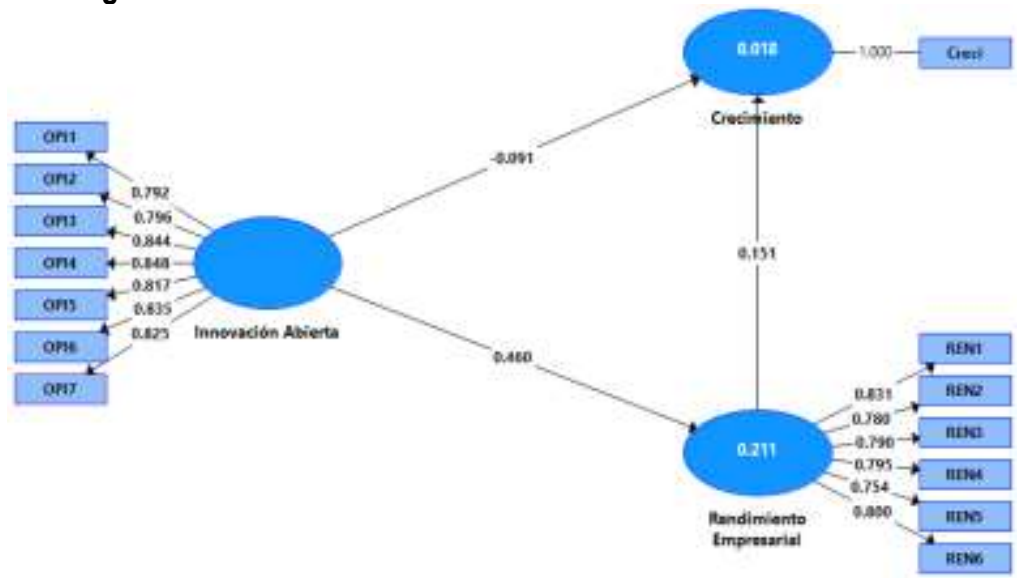

Fuente: Elaboración propia 
La Figura 1 indica, en primera instancia, que la totalidad de las cargas factoriales estandarizadas de la innovación abierta y el rendimiento empresarial son mayores al valor recomendado de 0.7 (Hair, Black, Babin y Anderson, 2014) y, en segunda instancia, la innovación abierta tiene efectos positivos significativos en el nivel del rendimiento empresarial $(\beta=0.460, p<0.001)$, el cual explica el $21.1 \%$ de la varianza del rendimiento empresarial $\left(R^{2}=\right.$ 0.211 ), como viene indicado por el valor en el círculo del rendimiento empresarial, mientras que parece poco probable que la innovación abierta tenga efectos positivos significativos en el nivel del crecimiento $(\beta=0.091, p<0.153)$, ya que sólo explica el $1.8 \%$ de la varianza del nivel de crecimiento $\left(R^{2}=0.018\right)$.

En este sentido, es posible establecer que la innovación abierta es un predictor moderadamente fuerte del rendimiento empresarial, mientras que este constructo no predice el nivel de ventas de manera directa, aunque sí lo hace indirectamente a través del rendimiento empresarial, ya que el rendimiento empresarial sí es un predictor moderadamente fuerte del nivel de ventas $(\beta=0.151, p<0.001)$, lo cual permite concluir que las dos relaciones path de la innovación abierta son estadísticamente significativas, de acuerdo a los resultados de las estimaciones de los parámetros de los tres constructos del modelo de innovación abierta que se presentan el la Figura 1 (Hair et al., 2014; Bentler y Huang, 2014).

Adicionalmente, los resultados obtenidos en este estudio empírico tienen distintas implicaciones tanto para los directivos como para las propias empresas, siendo una primera implicación la referente a que los datos derivados de la aplicación de las 460 encuestas, permitieron un análisis general de los efectos que tiene la innovación abierta en el nivel de crecimiento y el rendimiento empresarial en un sector en particular (la industria automotriz de México), por lo cual es posible establecer que las actividades de la innovación abierta son un factor determinante en el rendimiento empresarial, e indirectamente también determinan el nivel del crecimiento de las Pymes de la industria automotriz (Erol y Klug, 2020).

Una segunda implicación derivada de los resultados obtenidos es que la mayoría de los estudios publicados en la literatura científica, se han orientado en el análisis y discusión de la innovación abierta y el rendimiento financieros desde en contexto de las grandes empresas trasnacionales (Del Río et al., 2016; Kastelli et al., 2016; Seyfettinoglu, 2016), y son relativamente pocos los estudios que se han enfocado en el análisis de la innovación abierta, el nivel de crecimiento y el rendimiento empresarial desde la perspectiva de las Pymes manufactureras (Omta et al., 2014; Triguero et al., 2018), por lo cual es posible establecer que la relación existente entre los tres constructos puede considerarse como inconclusa.

Una tercera implicación de los resultados obtenidos es que la implementación de la innovación abierta, genera oportunidades para que las Pymes manufactureras puedan desarrollar actividades de colaboración con otras empresas del mismo sector o con organismos nacionales e internacionales, ya que la colaboración permite que las empresas no solamente compartan conocimiento y habilidades entre su personal, sino también disponer de los recursos de otras empresas y organismos para que las Pymes puedan incrementar significativamente su nivel de innovación (Dugdale y Strawn, 2017) y, como consecuencia de ello, mejorar su nivel de crecimiento y rendimiento empresarial.

Una cuarta implicación emanada de los resultados obtenidos es que la apertura de la infraestructura física para fomentar la interacción entre los trabajadores de las empresas y 
organismos participantes en las actividades de colaboración, los recursos y la información son tres de los pilares más importantes en los cuales descansan las actividades de la innovación abierta, ya que permite a las Pymes manufactureras, entre las que se destacan las que integran la industria automotriz, mejorar significativamente no solamente los niveles de educación y entrenamiento de su personal, sino también un mayor nivel de aprendizaje medioambiental (Skovgaard, Özkil y Mougaard, 2016; Erol, Jäger, Hold, Ott y Sihn, 2016), el cual se puede transformar en un mayor nivel de crecimiento y rendimiento empresarial.

Finalmente, una quinta y última implicación de los resultados obtenidos es que aun cuando se han confirmado la relación entre la innovación abierta y el rendimiento empresarial en un amplio número de estudios publicados en la literatura científica (Triguero et al., 2018), los resultados obtenidos en este estudio demuestran que esta relación es verdadera, pero solamente para las Pymes manufactureras, ya que el tamaño de las empresas es considerado como un factor fundamental que explica la adopción e implementación de las actividades de la innovación abierta (Sáez, Díaz y González, 2016). Sin embargo, para que las empresas tengan mayores posibilidades de implementar este tipo de actividades, requieren del trabajo conjunto con otras empresas y organizaciones, ya que por sí solas muchas de ellas no tendrían la posibilidad de adoptar la innovación abierta.

\section{Conclusiones}

Los resultados obtenidos en este estudio generan diversas conclusiones, entre las que destacan las tres siguientes. El modelo teórico de la relación existente entre la innovación abierta, el nivel de crecimiento y el rendimiento empresarial tiene una elevada consistencia interna, al generar una excelente correlación entre los tres constructos, ya que este modelo teórico contempla una visión general de las principales actividades de la innovación abierta existentes en la literatura científica (innovación abierta interna e innovación abierta externa), por lo cual es posible concluir la existencia de una relación estrecha entre las actividades internas y externas de la innovación abierta, el nivel de crecimiento y el rendimiento empresarial de las Pymes manufactureras.

Una segunda conclusión de los resultados obtenidos es que la innovación abierta permite a las Pymes manufactureras, esencialmente a las que integran la industria automotriz, no solamente compartir el conocimiento y las habilidades con sus principales socios comerciales, sino también la realización de actividades de colaboración con universidades y centros de investigación públicos y privados, lo cual facilita la adopción e implementación de las actividades de la innovación abierta. Sin embargo, las Pymes manufactureras tienen que realizar cambios 0 mejoras al interior de la organización, ya que la innovación abierta requiere del intercambio de conocimiento, habilidades y experiencias entre el personal de las distintas empresas e instituciones que colaboran.

Una tercera conclusión derivada de los resultados obtenidos es que generalmente una de las principales metas que tiene toda Pyme manufacturera, particularmente las que integran la industria automotriz, es mejorar significativamente su nivel de crecimiento y rendimiento empresarial. Así, para lograr esta meta es necesario que los directivos de las Pymes manufactureras en primera instancia, tengan entre sus estrategias empresariales la adopción e implementación de las actividades de la innovación abierta, ya que es precisamente a través de 
ella que se facilitará tanto la mejora del nivel del crecimiento como el incremento significativo del rendimiento empresarial.

En términos generales, se está incrementando cada vez más la importancia que tienen las actividades de la innovación abierta en las Pymes manufactureras, sobre todo en aquellas que integran la industria automotriz, ya que un número importante de Pymes son proveedoras de las grandes empresas armadoras de automóviles, por lo cual es necesario que adopten e implementen las actividades de la innovación abierta para que estén en posibilidades de compartir habilidades, conocimientos y recursos, logrando con este tipo de actividades de colaboración no solamente incrementar sus actividades de innovación, sino también mejorar su nivel de crecimiento y rendimiento empresarial, de ahí la importancia que tiene este estudio de aportar evidencia empírica de la relación existente entre estos tres importantes constructos.

\section{Referencias Bibliográficas}

Ahn, Joon Mo, Minshall, Tim y Mortara, Letizia. (2017). Understanding the human side of openness: The fit between open innovation models and CEP characteristics. R\&D Management. Volume 47, Numero 6, USA (Pp. 727-738).

Avalos Quispe, Grover Alfredo y Hernández Simon, Luis Manuel. (2019). Open innovation in SMEs: Potential and realized absorptive capacity for interorganizational learning in Dyad collaborations with academia. Journal of Open Innovation and Technology Marketing Complexity. Volume 5, Numero 3, Switzerland (Pp. 72-85).

Bag, Surajit. (2014). Impact of sustainable supply chain management on organizational performance: Mediating effects of leadership. Indian Journal of Management Science. Volume 4, Numero 3, India (Pp.10-25I).

Benneworth, Paul, Pinheiro, Rómulo y Karlsen, James. (2017). Strategic agency and institutional change: Investigating the role of universities in regional innovation systems (RISs). Regional Studies. Volume 5, Numero 2. U.K. (Pp. 235-248). http://dx.doi.org/10.1080/00343404.2016.1215599

Bentler, Peter y Huang, Wenjing. (2014). On components, latent variables, PLS, and simple methods: Reactions to Rigdon's rethinking of PLS. Long Range Planning. Volume 47, Numero 1 Switzerland (Pp. 136-145). http://dx.doi.org/10.1016/j.Irp.2014.02.005

Bogers, Marcel, Zobel, Ann Kristin, Afuah, Allan, Almiral, Esteve, Brunswicker, Sabine, Dahlander, Linus y Wal, Lars. (2017). The open innovation research landscape: Established perspectives and emerging themes across different levels of analysis. Industry and Innovation. Volume 24, Numero 1, UK (Pp. 8-18). http://dx.doi.org/10.1080/13662716.2016.1240068

Brunswicker, Sabine y Chesbrough, Henry. (2018). The adoption of open innovation in large firms. Research Technology Management. Volume 61, Numero 1. UK (Pp. 35-45). http://dx.doi.org/10.1080/08956308.2018.1399022

Brunswicker, Sabine y Ehrenmann, Frank. (2013). Managing open innovation in SMEs: A good practice example of a German software firm. International Journal of Industrial Engineering and Management. Volume 4, Numero 1, UK (Pp. 33-41).

Bryman, Alan. (2016). Social Research Methods. 5th Edition. Oxford University Press. UK. Cammarano, Aantonello, Caputo, Mauro, Lamberti, Emilia y Michelino, Francesca. (2017). Open innovation and intellectual property: A knowledge-based approach. Management 
Decision. Volume 55, Numero 9. United Kingdom (Pp. 1182-1195). http://dx.doi.org/10.1108/MD-03-2016-0203

Carneiro, Alberto. (2007). What is required for growth? Business Strategy Series. Volume 8, Numero 1. United Kingdom (Pp. 51-57). http://dx.doi.org/10.1108/17515630710686888 Chesbrough, Henry y Brunswicker, Sabine. (2014). A fad or a phenomenon? The adoption of open innovation practices in large firms. Responsible Technology Management. Volume 57, Numero 2, UK (Pp. 16-25).

de Oliveira, Lindomar Subtil, Echevestre Soares, María Elisa, Cortimiglia Nogueira, Marcelo y Gonçalves Colini, Giovani. (2017). Analysis of determinants for open innovation implementation in regional innovation systems. RAl Review Administrative e Innovation. Volume 14, Numero 2, Brasil (Pp. 119-129). http://dx.doi.org/10.1016/i.rai.2017.03.006

Del Río, Pablo, Peñasco, Cristina y Romero Jordan, Desiderio. (2016). What drives ecoinnovators? A critical review of the empirical literature based on econometric methods. Journal of Cleaner Production. Volume 112, Numero 1. United States (Pp. 2158-2170). http://dx.doi.org/10.1016/j.jclepro.2015.09.009

Dijkstra, Theo y Henseler, Jörg. (2015a). Consistent and asymptotically normal PLS estimators for linear structural equations. Computational Statistics and Data Analysis. Volume 81, Numero 1. Germany (Pp. 10-23). http://dx.doi.org/10.1016/i.csda.2014.07.008

Dijkstra, Theo y Henseler, Jörg. (2015b). Consistent partial least squares path modeling. MIS Quarterly. Volume 39, Numero 2, USA (Pp. 297-316). http://dx.doi.org/10.25300/MISQ/2015/39.2.02

Dijkstra, Theo. (2014). PLS' Janus face - Response to Professor Rigdon's rethinking partial least squares modeling: In praise of simple methods. Long Range Planning. Volume 47, Numero 1. Switzerland (Pp. 146-153). http://dx.doi.org/10.1016/j.Irp.2014.02.004

do Valle Oom, Patricia y Assaker, Guy. (2015). Using partial least squares structural equation modeling in tourism research: A review of past research and recommendations for future applications. Journal of Travel Research. Volume 55, Numero 6, USA (Pp. 795-708). http://dx.doi.org/10.1177/0047287515569779

Dodourova, Mariana y Bevis, Keith. (2014). Networking innovation in the European car industry: Does the open innovation model fit? Transportation Research Part A: Policy and Practice. Volume 69, Numero 2, United Kingdom (Pp. 252-271). http://dx.doi.org/10.1016/.tra.2014.08.021

Dugdale, Shirley y Strawn, Brian. (2017). Crafting an innovation landscape. Planning for Higher Education. Volume 7, Numero 1. United States (Pp. 10-19).

Erol, Selim y Klug, Siegrun. (2020). Together we are less alone: A concept for a regional open innovation-learning lab. Procedia Manufacturing. Volume 45, Numero 4, United States (Pp. 540-545).

Erol, Selim, Jäger, Aandreas, Hold, Philipp, Ott, Karl y Sinn, Wifried. (2016). Tangible industry 4.0: A scenario-based approach to learning for the future of production. Procedia CIRP. Volume 54, Numero 9, UK (Pp. 13-18). http://dx.doi.org/10.1016/j.procir.2016.03.162 European Commission (2014). The Future of Open Innovation. European Commission. Belgica. 
European Commission (2016). Open Innovation, Open Science, Open to the World: A Vision for Europe. Directorate-General for Research and Innovation. Belgica.

Forza, Cipriano. (2016). Surveys. In Christer Karlsson (Ed.), Research Methods for Operations Management. Routledge. USA.

Hair, Joseph, Black, William, Babin, Barry y Anderson, Rolph. (2014). Multivariate Data Analysis. 7th Edition. Harlow, Pearson Education. UK.

Hair, Joseph, Celsi, Mary, Money, Arthur, Samouel, Phillip y Page, Michael. (2016). Essentials of Business Research Methods. 3rd Edition. Routledge, USA.

Heidemann, Astrid y Timenes, Bjorge. (2017). Open innovation: On the influence of internal and external collaboration on degree of newness. Business Process Management Journal. Volume 23, Numero 6, UK (Pp. 1129-1143).

Henseler, Jörg, Ringle, Christian y Sarstedt, Marko. (2015). A new creation for assessing discriminant validity in variance-based structural equation modeling. Journal of the Academy of Marketing Science. Volume 43, Numero 1, Switzerland (Pp. 115-135). http://dx.doi.org/10.1007/s11747-014-0403-8

$\mathrm{Hu}$, Yansong, McNamara, Peter y McLoughlin, Damien. (2015). Outbound open innovation in bio-pharmaceutical out-licensing. Technovation. Volume 35, Numero 1, United Kingdom (Pp. 46-58). http://dx.doi.org/10.1016/j.technovation.2014.07.004

Hungund, Sumukh y Kiran, Karl. (2015). Open Innovation Practices and Challenges Among Indian SMEs. Springer. India.

Kastelli, loanna, Tsakanikas, Anggelos y Caloghirou, Yannis. (2016). Technology transfer as a mechanism for dynamic transformation in the food sector. Journal of Technology Transfer. Volume 8, Numero 1, Switzerland (Pp. 1-19). http://dx.doi.org/10.1007/s10961016-9530-3

Kratzer, Jan, Meissner, Dirk y Rould, Vitaly. (2017). Open innovation and company culture: Internal openness makes the difference. Technological Forecasting and Social Change. Volume 119, Numero 1, United States (Pp. 128-138). http://dx.doi.org/10.1016/i.techfore.2017.03.022

Kruger, Carl y Johnson, Roy. (2009). Assessment of knowledge management growth: A South Africa perspective. Aslib Proceedings, New Information Perspectives. Volume 61, Numero 6, Germany (Pp. 542-564). http://dx.doi.org/10.1108/00012530911005517

Linder, Jane. (2006). Does innovation drive profitable growth? New metrics for a complete picture. Journal of Business Strategy. Volume 27, Numero 5, United States (Pp. 3844). http://dx.doi.org/10.1108/02756660610692699

Lopes, Ana Paula y Carvalho, Marly. (2018). Evolution of the open innovation paradigm: Towards a contingent conceptual model. Technological Forecasting and Social Change. Volume 132, Numero 2, United States (Pp. 284-298). http://dx.doi.org/10.1016/j.techfore.2018.02.014

Maier, Andreea, Keppler, Timo y Maier, Dorin. (2014). Innovation the new trend in today's challenging economy. In The Bucharest University of Economic Studies (Ed.), The 13 International Conference on Informatics in Economy, Education, Research \& Business Technologies. Bucharest, Romania. (Pp. 15-18).

Martinez Conesa, Isabel, Soto Acosta, Pedro y Carayannis, Elias George. (2017). On the path towards open innovation: Assessing the role of knowledge management capability and 
environmental dynamism in SMEs. Journal of Knowledge Management. Volume 21, Numero 4, Italy (Pp. 553-565). http://dx.doi.org/10.1108/JKM-09-2016-0403

Omta, Onno, Fortuin, Frank y Dijkman, Nicole. (2014). Open innovation in the food industry: An evidence based guide. Food Valuation. Volume 10, Numero 1, Holanda (Pp. 1-11).

Organización para la Cooperación y el Desarrollo Económico, OECD (2005). Oslo Manual: Guidelines for collecting and interpreting innovation data. In OECD (Ed.), The Measurement of Scientific and Technological Activities. OECD Publishing. Francia. Pellegrini, Luisa, Lazaretto, Valentina y Manzini, Raffaella. (2014). Open innovation in the food and drink industry. Journal of Agricultural Food Industry Organization. Volume 12, Numero 1, Germany (Pp. 75-94). http://dx.doi.org/10.1515/jafio-2013-0023

Podmetina, Daria, Teplov, Roman, Albats, Ekaterina y Dabrowska, Justyna. (2016). Perception vs. reality: The adoption of open innovation in European companies. Academic Management Proceedings. Volume 20, Numero 1, USA (Pp. 177-199). http://dx.doi.org/10.5465/ambpp.2016.17799abstract

Randhawa, Krithinka, Wilden, Ralf y Hohberger, Jan. (2016). A bibliometric review of open innovation: Setting a research agenda. Journal of Product Innovation Management. Volume 37, Numero 3, USA (Pp. 750-765). http://dx.doi.org/10.1111/jpim.12312

Richter, N.F., Cepeda Carrion Gabriel, Roldán Salgueiro Jose Luis y Ringle, Christian. (2016). European management research using partial least squares structural equation modeling (PLS-SEM). European Management Journal. Volume 34, Numero 6, Belgica (Pp. 589597). http://dx.doi.org/10.1016/i.emj.2016.08.001

Richter, Nicole, Sinkovics, Rudolf, Ringle, Christian y Schlägel, Christopher. (2016). A critical look at the use of SEM in international business research. International Marketing Review. Volume 33, Numero 3, United Kingdom (Pp. 376-404). http://dx.doi.org/10.1108/IMR-04-2014-0148

Rivotti, Miriam. (2015). Kick-starting innovation: A fast-track version of innovation scoring for start-ups. Dissertation submitted as partial requirement for the degree of Master of Science in Management. ISCTE Business School. Portugal.

Sáez Martínez, Francisco, Díaz García, Cristina y González Moreno, Angela. (2016). Factors promoting environmental responsibility in European SMEs: The effect of performance. Sustainability. Volume 8, Numero 9, Switzerland (Pp. 898-911). http://dx.doi.org/10.3390/su8090898

Sag, Serhat, Sezen, Büllent y Güzel, Mevlüdiye. (2016). Factors that motivate or prevent open innovation by SMEs in developing countries and policy suggestions. Procedia - Social and Behavioral Sciences. Volume 235, Numero 6, United Kingdom (Pp. 756-763).

Sánchez, Gabriel, Trinchera, Luis y Russolillo, Gabriela. (2015). R Package PLSPM: Tools for Partial Least Squares Path Modeling (PLS-PM) Version 0.4.7. Computer Software. España.

Schiuma, Giovanni y Carlucci, Daniela. (2018). Managing strategic partnership with universities in innovation ecosystems: A research agenda. Journal of Open Innovation and Technology Marketing Complexity. Volume 4, Numero 3, Switzerland (Pp. 25-35). http://dx.doi.org/10.3390/joitmc4030025 
Seyfettinoglu, Umit. (2016). Analysis of relationship between firm performance and open innovation strategies and stages in the Turkish food and beverage industry. New Mediterranean. Volume 15, Numero 1, United Kingdom (Pp. 42-52).

Sivam, Ashwin, Dieguez, Teresa, Pinto, Luis y Silva, Fernando. (2019). Key setting for successful open innovation arena. Journal of Computational Design and Engineering. Volume 6, Numero 4, United Kingdom (Pp. 507-515). http://dx.doi.org/10.1016/j.jcde.2019.03.005

Skovgaard, Jensen, Özkil, Ali Gürcan y Mougaard, Krestine. (2016). Makerspaces in engineering education: A case study. Paper presented at the ASME 2016: International Design Engineering Technical Conference and Information in Engineering Conference. London, 2016. http://dx.doi.org/10.1115/DETC2016-60066

Spithoven, André, Vanhaverbeke, Wim y Roijakkers, Nadine. (2013). Open innovation practices in SMEs and large enterprises. Small Business Economics. Volume 41, Numero 3, Switzerland (Pp. 537-562). http://dx.doi.org/10.1007/s11187-012-9453-9

Triguero, Angela, Fernández, Sara y Sáez Martinez, Francisco Javier. (2018). Inbound open innovation strategies and eco-innovation in the Spanish food and beverage industry. Sustainable Production and Consumption. Volume 15, Numero 1, United Kingdom (Pp. 49-64). http://dx.doi.org/10.1016/i.spc.2018.04.002

Van de Vrande, Vareska, De Jong, Jeroen, Vanhaverbeke, Wim y De Rochemont, Maurice. (2009). Open innovation in SMEs: Trends, motives and management challenges. Technovation. Volume 29, Numero 6/7, United Kingdom (Pp. 423-437). http://dx.doi.org/10.1016/i.technovation.2008.10.001

Vather, Priit, Love, James y Roper, Stephen. (2014). Openness and innovation performance: Are small firma different? Industry and Innovation. Volume 21, Numero $7 / 8$, United Kingdom (Pp. 553-573).

Wang, Ding, Dunn, Nick y Coulton, Paul. (2015). Grassroots Make Spaces: A Recipe for Innovation? EAD. Francia. 